\title{
Combination of hydrotropic nicotinamide with nanoparticles for enhancing tacrolimus percutaneous delivery
}

This article was published in the following Dove Press journal:

International Journal of Nanomedicine

19 August 2016

Number of times this article has been viewed

\author{
Wenhui Pan \\ Mengyao Qin \\ Guoguang Zhang \\ Yueming Long \\ Wenyi Ruan \\ jingtong Pan \\ Zushuai Wu \\ Tao Wan \\ Chuanbin Wu \\ Yuehong $\mathrm{Xu}$ \\ Department of Pharmaceutics, School \\ of Pharmaceutical Sciences, Sun Yat- \\ sen University, Guangzhou, People's \\ Republic of China
}

Correspondence: Yuehong $\mathrm{Xu}$ Department of Pharmaceutics, School of Pharmaceutical Sciences, Sun Yat-sen University, No 132, Out-ring East Road, University Town, Guangzhou 510006, People's Republic of China

Tel/fax +862039943119

Email Issxyh@mail.sysu.edu.cn
Abstract: Tacrolimus (FK506), an effective immunosuppressant for treating inflammatory skin diseases, hardly penetrates into and through the skin owing to its high hydrophobicity and molecular weight. The aim of this study was to develop a hybrid system based on nicotinamide (NIC) and nanoparticles (NPs) encapsulating FK506, such as FK506-NPs-NIC, for facilitating percutaneous delivery, which exploited virtues of both NIC and NPs to obtain the synergetic effect. Solubility and percutaneous permeation studies were carried out. The results showed that NIC could increase the solubility and permeability of FK506 and that $20 \%$ (w/v) NIC presented higher FK506 permeability and was thus chosen as the hydrotropic solution to solubilize FK506 and prepare FK506-NPs-NIC. Hyaluronic acid (HA) was chemically conjugated with cholesterol (Chol) to obtain amphiphilic conjugate of HA-Chol, which self-assembled NPs in $20 \%$ NIC solution containing FK506. The particle size, zeta potential, and morphology of NPs were characterized. The encapsulation efficiency and in vitro percutaneous permeation of NPs were evaluated in the presence and absence of NIC. The results demonstrated that hydrotropic solubilizing FK506 was readily encapsulated into NPs with a higher encapsulation efficiency of $79.2 \% \pm 4.2 \%$, and the combination of NPs with NIC exhibited a significantly synergistic effect on FK506 deposition within the skin $\left(2.39 \pm 0.53 \mu \mathrm{g} / \mathrm{cm}^{2}\right)$ and penetration through the skin $\left(13.38 \pm 2.26 \mu \mathrm{g} / \mathrm{cm}^{2}\right)$. The effect of the combination of NPs with NIC on drug permeation was further visualized by confocal laser scanning microscope through in vivo permeation studies, and the results confirmed that NPs-NIC synergistically enhanced the permeation of the drug into the skin. The cellular uptake performed in $\mathrm{HaCaT}$ cells presented a promoting effect of NPs on cellular uptake. These overall results demonstrated that HA-Chol-NPs-NIC can synergistically improve the percutaneous delivery of FK506, and it is a novel potential strategy based on a nano-sized carrier for FK506 to treat skin diseases.

Keywords: tacrolimus, nicotinamide, hyaluronic acid, nanoparticles, percutaneous delivery

\section{Introduction}

Tacrolimus (FK506), a strong immunosuppressant drug, was isolated from Streptomyces tsukubaensis. The mechanism of FK506 was similar to that of cyclosporine A but $\sim 30-100$-fold in vitro and 10-20-fold in vivo higher than cyclosporine A for immunosuppressant activity. ${ }^{1}$ Topical administration of FK506 was effective in the treatment of various inflammatory skin diseases, including atopic dermatitis (AD), ${ }^{2,3}$ psoriasis, ${ }^{4}$ and eczema. ${ }^{5}$ The main target of FK506 was the dermis with its lymphocytes. ${ }^{6}$ However, FK506 hardly penetrated into and through the skin for ensuring the effective therapeutic level at the diseased site because of its high hydrophobicity and high molecular weight (MW 822.05 Da). The commercial ointment in the international submit your manuscript Dovepress 15 in 0 
market, Protopic ${ }^{\circledR}$ (Astellas Toyama Co., Ltd., Toyama, Japan), was formulated with propylene carbonate to dissolve FK506 and greasy vehicle, which did not ensure adequate drug delivery to the disease site. ${ }^{7}$ The formulation for FK506 percutaneous delivery remained a challenge. With the aim of improving percutaneous permeation, various approaches based on colloidal vesicles have been developed for FK506 dermal delivery. Microemulsion, ${ }^{6}$ ethosomes, ${ }^{8}$ lipid nanoparticles (NPs), ${ }^{9}$ and modified nanolipid carrier ${ }^{10}$ have been addressed, and they all presented enhanced FK506 percutaneous permeation. However, each of these approaches has its respective challenges in terms of safety, feasibility, or stability, for example, considerable surfactants for microemulsion, hot homogenization preparation for lipid NPs and modified nanolipid carrier, and lecithin stability for ethosomes. Among these nanocarriers, NPs possessed the ability to load hydrophobic drugs and adhere to the surface of the skin providing an occlusive effect, thus enhancing the hydration of the skin, ${ }^{11}$ or to be held in the lipid matrix of stratum corneum (SC) and in hair follicles, ${ }^{12}$ which facilitated greater drug permeation of the skin and the forming of a drug reservoir at the site of action in the skin. ${ }^{13}$ Therefore, NPs have shown promise in topical drug delivery, and the most commonly used NPs for topical drug delivery were biodegradable polymeric NPs with respect to safety. In this study, NPs were constructed with natural hyaluronic acid (HA) and cholesterol (Chol), ensuring the safety and stability, and the NPs were formed by self-assembling without heating.

HA, a component of extracellular matrix and synovial fluids, is a naturally linear polysaccharide consisting of repeating units of $N$-acetyl glucosamine and glucuronic acid. ${ }^{14}$ It possesses advantageous properties such as viscoelasticity, biocompatibility, hydration, and lubrication and is widely used in foods, cosmetics, and medical surgeries. Balazs $^{15}$ and Gaffney et $\mathrm{al}^{16}$ evaluated its application in dermal delivery of anticancer drugs. The enhancement of HA on nonsteroidal anti-inflammatory diclofenac percutaneous permeation was also evaluated, and HA could increase the partition, deposition, and localization of diclofenac in the skin. ${ }^{17,18}$ However, the exact mechanism of HA-mediated skin permeation was not clearly demonstrated. Many reports discussed the factors as follows. First, the HA receptor of CD44 distributed in skin might assist the active transport of HA or the cotransport of HA and active agents. ${ }^{17,19}$ Second, HA was a hydrophilic macromolecule and could hydrate the skin, resulting in enhancement of drug absorption and retention. Third, the hydrophobic chain of HA could enhance its permeability across the SC. ${ }^{17,20,21}$ HA was also widely used as a ligand to modify delivery systems such as NPs (to deliver drugs to target tumor cells), owing to the specific interaction between HA and CD44 receptors, which overexpressed on the surface of some tumor cells, and HA has been widely prepared to be self-assembling NPs and investigated as carriers for receptor-mediated drug targeting in cancer therapy. ${ }^{22}$ The vital of HA-based self-assembling NPs was choosing a hydrophobic molecule to be conjugated to HA to prepare an amphiphilic HA derivative, which self-assembled into nanosized particles consisting of a hydrophobic core surrounded by the hydrophilic HA shell, ${ }^{23}$ and the hydrophobic drugs would be encapsulated in the core of NPs through hydrophobic interaction. The hydrophobic moieties with low or high MW, tetradecylamine, 5b-colanic acid, monofunctional or biofunctional fatty amines, or multiple nitrogen-containing derivatives such as poly(L-lysine) and polyethyleneimine, ceramide, and Chol have been reported to be linked with the carboxylic acid group of HA through the essentially mild conditions of the carbodiimide chemistry. ${ }^{23} \mathrm{HA}-\mathrm{Chol}-\mathrm{NPs}$ have been investigated to encapsulate chemotherapeutic drug docetaxel (DTX) or 2b protein/siRNA complex for targeting delivery drugs to tumor cells. ${ }^{24,25}$ However, the application of NPs based on HA-Chol for targeting delivery drugs to the skin has not been evaluated so far.

For preparation of hydrophobic drug-loaded NPs, the drug solubility was vital in achieving enhanced drug entrapment and high stability of NPs. ${ }^{26}$ Therefore, increasing the drug solubility prior to NPs preparation should be considered. Nicotinamide (NIC), a water-soluble vitamin $\left(\mathrm{B}_{3}\right)$, was commonly used as a hydrotropic agent due to its ability to augment the solubility of some poorly water-soluble molecules. ${ }^{27-29}$ Moreover, NIC presented enhancing DTX permeation through rat skin after DTX was solubilized by NIC to form DTX-NIC complex and then prepared to be nanostructured lipid carriers. ${ }^{30}$ In the cosmetic industry, NIC was widely used in topical preparations due to its moisturizing and depigmenting properties. NIC was an inhibitor of PARP-1. PARP regulated the expression of immunomodulatory protein, major histocompatibility complex class II, and NF-kB. ${ }^{31}$ Consequently, NIC facilitated the treatment of some inflammatory skin diseases, including bullous pemphigoid, acne, rosacea, and AD. ${ }^{32,33}$ NIC was also reported to enhance the efficacy of calcipotriene for treating psoriasis when they were used in combination. ${ }^{34}$ The benefits of NIC to the skin made it a potentially attractive vehicle for topical formulations. In this project, NIC was hypothesized to solubilize FK506 and exert positive effects on FK506-NPs formulation, permeation, and efficacy. 
On the basis of the abovementioned considerations, with the aim to establish an effective percutaneous delivery system for FK506 to treat skin diseases, a combined strategy for FK506 based on NIC and HA-Chol-NPs has been developed, which is expected to exploit both virtues of NIC and NPs to obtain synergetic effects. The effects of NIC on solubility and permeability of FK506 were evaluated. An amphiphilic conjugate HA-Chol was synthesized by hydrophobically modifying HA backbone with Chol, and FK506-HA-CholNPs-NIC/HA-Chol-NPs self-assembled in NIC solution or aqueous solution. The particle size, zeta potential, morphology, and encapsulation efficiency (EE) were characterized. In vitro permeability of FK506 from HA-Chol-NPs-NIC was evaluated in comparison with HA-Chol-NPs, NIC complex solution, aqueous suspension, and commercial preparation of ointment (Protopic), respectively. The effect of the combination of NIC with NPs on drug retention in the skin was further visualized with confocal laser scanning microscope (CLSM) through in vivo permeation studies. Finally, the immortal human keratinocytes ( $\mathrm{HaCaT}$ ) were employed to evaluate the cellular uptake of FK506-NPs-NIC in comparison with FK506-NPs, FK506-NIC complex solution, and FK506 aqueous suspension. The graphical summary is provided in Figure S1.

\section{Materials and methods Materials}

Cholesteryl chloroformate, $N$-(3-dimethylaminopropyl)- $N$ 'ethylcarbodiimide hydrochloride (EDC), $N$-hydroxysuccinimide, and pyrene were purchased from Aladdin (Shanghai, People's Republic of China). Sodium hyaluronate (HA, MW $30 \mathrm{kDa}$ ) was obtained from Bloomage Freda Biopharm Co., Ltd. (Shandong, People's Republic of China). Anhydrous ethylenediamine was purchased from Damao Chemical Reagent Factory (Tianjin, People's Republic of China). Trimethylamine was obtained from Tianjin Zhiyuan Chemical Reagent Co., Ltd. (Tianjin, People's Republic of China). NIC was a gift of Guangzhou Changlong Technology Co., Ltd. (Guangzhou, People's Republic of China). FK506 was purchased from Teva Czech Industries (Opava-Komarov, Czech Republic). The 502 super glue was purchased from Henggu Plastic Industry Co., Ltd. (Zhejiang, People's Republic of China). Coumarin 6 (C6); penicillin; 4',6-diamidino-2phenylindole (DAPI); and streptomycin were obtained from Sigma-Aldrich Co. (St Louis, MO, USA). Fetal bovine serum (FBS) and Dulbecco's Modified Eagle's Medium (DMEM) were purchased from Thermo Fisher Scientific (Waltham, MA, USA). Other reagents were of analytical grade.

\section{Influence of NIC on FK506}

\section{Solubility}

The influence of NIC on FK506 solubility was determined using the 24-hour shake flask method. ${ }^{35}$ In brief, excess FK506 was added to screw-capped vials containing a fixed volume of NIC solution with different concentrations of $0 \%(\mathrm{w} / \mathrm{v}), 10 \%(\mathrm{w} / \mathrm{v}), 20 \%(\mathrm{w} / \mathrm{v})$, and $30 \%(\mathrm{w} / \mathrm{v})$. The suspension was maintained in a thermostatic shaker (THZ82BA; Jinchang Instrument Company, Jinchang, People's Republic of China) for 3 days at $100 \mathrm{rpm}$ and $37^{\circ} \mathrm{C}$ to reach the dissolution equilibrium. Aliquots from each vial were sampled and filtered through a $0.22 \mu \mathrm{m}$ microporous nylon membrane. The filtered aliquot was analyzed by highperformance liquid chromatography (HPLC).

\section{Permeability}

To determine the influence of NIC on FK506 permeability, in vitro permeation experiments were conducted. Sprague Dawley rats (male, 180-220 g) were purchased from the Experiment Animal Center of Sun Yat-sen University (Guangzhou, People's Republic of China). All work performed with animals were in accordance with the Principles of Laboratory Animal Care and Use in Research published by the Chinese Ministry of Health, and the protocols were approved by the Institutional Animal Care and Use Committee at Sun Yat-sen University. The Sprague Dawley rats were anesthetized and sacrificed by cervical dislocation, and the hair on the abdomen was carefully removed with a razor. The abdominal skin was excised, and the subcutaneous tissues were surgically removed. Then the skin was rinsed with normal saline. In vitro permeation was performed with vertical Franz diffusion cell under occlusive conditions with an effective contact area of $3.14 \mathrm{~cm}^{2}$ and receptor volume of $7.5 \mathrm{~mL}$ (TK-12A; Kaikai Technology Co., Ltd., Shanghai, People's Republic of China) according to our previous report. ${ }^{36}$ Skin was mounted on the receptor cell with SC facing the donor cell, and the receptor cell was filled with normal saline containing $25 \%$ ethanol as the receptor medium with respect to the sink condition. The skin temperature was maintained at $32^{\circ} \mathrm{C} \pm 1^{\circ} \mathrm{C}$ with a circulating water bath and stirred at $250 \mathrm{rpm}$. In all, $500 \mu \mathrm{L}$ of FK506 suspension $(0.1 \%, \mathrm{w} / \mathrm{v})$ containing $0 \%(\mathrm{w} / \mathrm{v}), 10 \%(\mathrm{w} / \mathrm{v})$, $20 \%(\mathrm{w} / \mathrm{v})$, and $30 \%(\mathrm{w} / \mathrm{v})$ NIC was applied onto the skin, respectively. In our preliminary experiment, FK506 in the receptor medium could not be detected until 10 hours of permeation in the groups with NIC and 12 hours of permeation in the group without NIC; thus, the receptor medium at intervals of 12 hours, 14 hours, 16 hours, 18 hours, 
20 hours, and 24 hours after permeation were sampled and analyzed by HPLC.

After 24 hours of permeation, the residual formulations were carefully removed from the skin and the skin was cut into pieces. Two volumes of methanol was added to the skin pieces for extracting FK506 retained in the skin. The extraction was performed by sonicating for 30 minutes following centrifuging for 5 minutes at 5,000 rpm. The supernatant was filtered with $0.22 \mu \mathrm{m}$ microporous nylon membrane and analyzed by HPLC.

\section{Synthesis of HA-Chol conjugates}

HA-Chol conjugates were prepared as shown in the schematic of Figure 1. In brief, anhydrous ethylenediamine $(3.34 \mathrm{~mL})$ and trimethylamine $(300 \mu \mathrm{L})$ were mixed with anhydrous methylene chloride $\left(\mathrm{CH}_{2} \mathrm{Cl}_{2} ; 30 \mathrm{~mL}\right)$, and
A

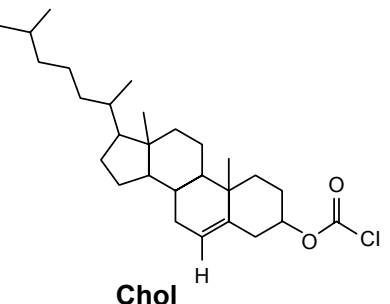

Chol $^{\mathrm{H}}$

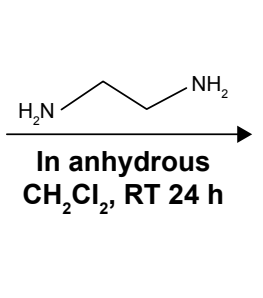<smiles>CC(C)CCCC(C)C1CCC2C3CCC4(CCC(OC(=O)NCCN)CC4CC23)C1</smiles>

Chol-Am

$\mathrm{H}$

EDC/NHS in $\mathrm{H}_{2} \mathrm{O} / \mathrm{DMF}(1 / 3, \mathrm{v} / \mathrm{v})$,

$48 \mathrm{~h}$

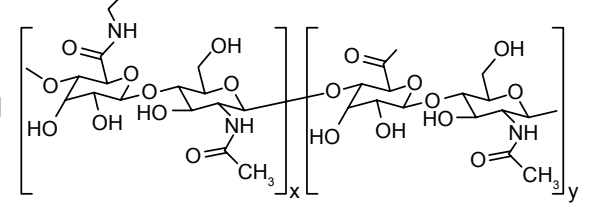

B

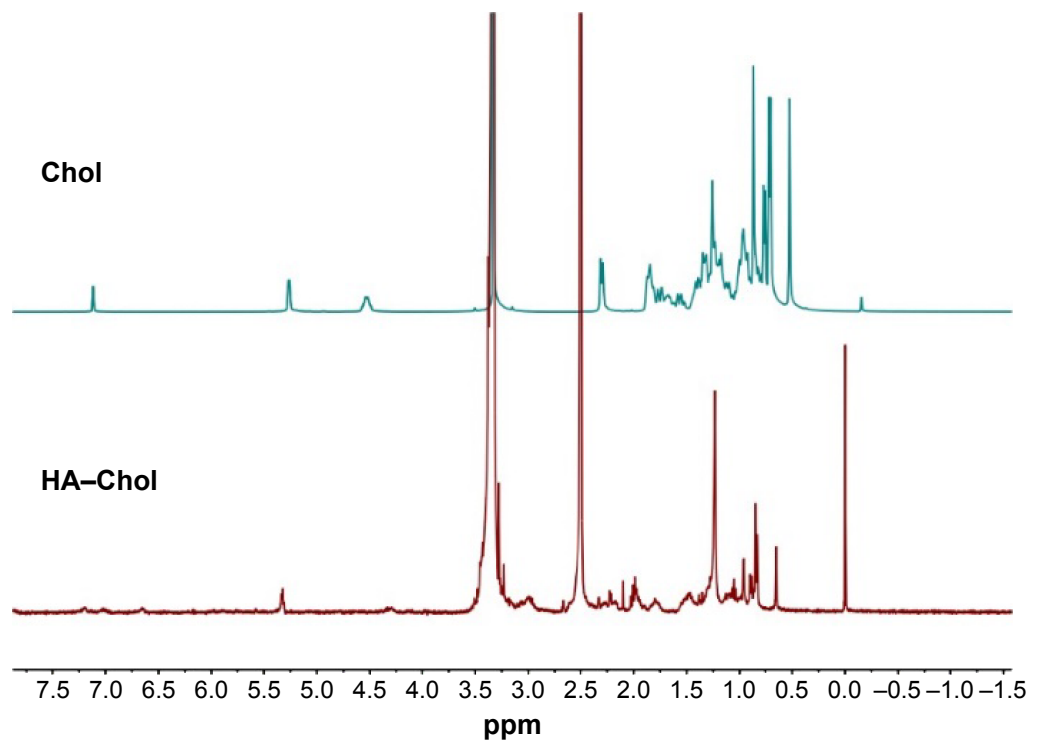

Figure I Synthesis of HA-Chol conjugation and its identification through NMR.

Note: (A) Synthetic scheme of HA-Chol. (B) 'H NMR spectrum of Chol and HA-Chol conjugates. HA-Chol was dissolved in DMSO-d6 and analyzed by 'H NMR using Bruker Avance III $400 \mathrm{MHz}$ NMR.

Abbreviations: Chol, cholesterol; Chol-Am, cholesteryl amine; $\mathrm{CH}_{2} \mathrm{Cl}_{2}$, methylene chloride; DMF, N,N-dimethylformamide; DMSO- $\mathrm{d}_{6}$, dimethyl sulfoxide- $\mathrm{d}_{6}$; EDC, I-(3dimethylaminopropyl)-3-ethylcarbodiimide; h, hours; HA, hyaluronic acid; HA-Chol, hyaluronic acid-cholesterol conjugates; NHS, N-hydroxysuccinimide; NMR, nuclear magnetic resonance; RT, room temperature. 
cholesteryl chloroformate dissolved in anhydrous $\mathrm{CH}_{2} \mathrm{Cl}_{2}$ $(10 \mathrm{~mL})$ was added drop wise to this solution under nitrogen stream. After stirring for 24 hours under nitrogen stream at room temperature, the solution was washed with distilled water for three times. After rotary evaporation, crude products were obtained. The crude products were dissolved in $\mathrm{CH}_{2} \mathrm{Cl}_{2}$ : methanol $(1: 1, \mathrm{v} / \mathrm{v})$ and filtered with $0.22 \mu \mathrm{m}$ microporous nylon membrane to remove biscarbamate, and the filtrate was rotary evaporated to obtain cholesteryl amine (Chol-Am). To chemically modify HA with Chol-Am, HA (1 g), EDC $(0.5 \mathrm{~g})$, and $N$-hydroxysuccinimide $(0.3 \mathrm{~g})$ were dissolved in distilled water: $N, N$-dimethylformamide $(1: 3, \mathrm{v} / \mathrm{v})$ and stirred for 2.5 hours to activate the carboxyl group of HA. After activation, Chol-Am (0.2-0.8 g) dissolved in $N, N$ dimethylformamide $(80 \mathrm{~mL})$ was added drop wise to the activated HA solution. After stirring for 48 hours, the solution was dialyzed against distilled water:tetrahydrofuran $(1: 1, \mathrm{v} / \mathrm{v})$ solution and water, respectively. HA-Chol conjugates with different degrees of substitution (DSs) were obtained following freeze-drying. DS was the number of Chol molecule per 100 sugar residues of $\mathrm{HA}$ determined by ${ }^{1} \mathrm{H}$ nuclear magnetic resonance (NMR). HA-Chol was dissolved in dimethyl sulfoxide- $\mathrm{d}_{6}$ and analyzed by ${ }^{1} \mathrm{H}$ NMR (400 MHz, Avance III; Bruker Optik GmbH, Ettlingen, Germany).

The critical aggregation concentration (CAC) was measured using an automatic microplate reader (Flex Station 3; Molecular Devices LLC, Sunnyvale, CA, USA) with pyrene as a hydrophobic probe. In all, $20 \mu \mathrm{L}$ of pyrene solution $(40 \mu \mathrm{g} / \mathrm{mL})$ in acetone was added to a tube. After evaporating acetone under a gentle nitrogen stream for 1 hour at room temperature, $4 \mathrm{~mL}$ of $\mathrm{HA}-\mathrm{Chol}$ solution with the concentration ranging from $0.1 \mu \mathrm{g} / \mathrm{mL}$ to $0.8 \mathrm{mg} / \mathrm{mL}$ was added to each tube to maintain the final concentration of pyrene at $1.0 \times 10^{-6} \mathrm{~mol} / \mathrm{L}$. The solution was sonicated for 10 minutes and vibrated under vapor bath overnight prior to determination of the fluorescence intensity. The fluorescence intensity was measured using the automatic microplate reader, and the emission intensities of $388 \mathrm{~nm}\left(I_{388}\right)$ and $375 \mathrm{~nm}\left(I_{375}\right)$ were obtained at a fixed excitation wavelength of $350 \mathrm{~nm}$. The CAC was determined by plotting the fluorescence intensity ratio of $I_{388} / I_{375}$ to the concentration of HA-Chol conjugates.

\section{Preparation and characterization of FK506-loaded NPs}

FK506-loaded NPs with NIC or without NIC were prepared with ultrasonic cell disruptor (JY92-2D; Ningbo Xinzhi Science and Technology Co., Ningbo, People's Republic of China). In brief, HA-Chol conjugates and FK506 were dissolved in $20 \%(\mathrm{w} / \mathrm{v})$ NIC solution or aqueous solution with the final FK506 concentration of $1 \mathrm{mg} / \mathrm{mL}$. After sonicating for 6 minutes, FK506-NP suspension was obtained.

The NP suspension was filtered with a $0.45 \mu \mathrm{m}$ microporous nylon membrane, and the particle size, polydispersity index, and zeta potential were determined using a Zetasizer Nano ZS (Malvern Instruments, Malvern, UK). The morphology of NPs was observed with atomic force microscope (AFM; Dimension FastScan; Bruker Optik GmbH). The NP suspension was dropped onto the surface of mica and dried at room temperature. The images were obtained using ScanAsyst mode and with a probe of FastScan-A/C. The analysis of the AFM images was processed with the NanoScope software Version 1.40 (Bruker Nano Surfaces Division, Santa Barbara, CA, USA).

EE of NPs was determined by gel permeation chromatography. In all, $500 \mu \mathrm{L}$ of NP suspension was added into the top of Sephadex LH-20 column. A total of $20 \mu \mathrm{L}$ of water was used to elute NPs and $20 \mathrm{~mL}$ of ethanol to elute free FK506. The effluent of ethanol was collected and determined by HPLC. The EE (\%) of FK506 was calculated according to the following equation:

$$
\mathrm{EE}(\%)=\frac{\text { Mass of FK506 incorporated in NPs }(\mathrm{mg})}{\text { Mass of FK506 introduced }(\mathrm{mg})} \times 100
$$

\section{In vitro permeation of HA-Chol-NPs- $\mathrm{NIC}$ and HA-Chol-NPs}

In all, $500 \mu \mathrm{L}$ of FK506-HA-Chol-NPs-NIC, HA-CholNPs, FK506-NIC complex suspension (with an equal amount of NIC to that of HA-Chol-NIC, 20\%, w/v), aqueous suspension, and $0.5 \mathrm{~g}$ of commercial preparation of Protopic with an equal amount of FK506 $(1 \mathrm{mg} / \mathrm{mL})$ were used in in vitro permeation experiments, and the procedure was the same as described in the Permeability section.

\section{In vivo permeation of C6-loaded NPs}

The effects of NIC, NPs, and NPs-NIC on the permeation in the skin were further evaluated visually with CLSM (Zeiss LSM 710; Carl Zeiss Meditec AG, Jena, Germany), and a hydrophobic fluorescent, C6, was used as the probe. Sprague Dawley rats were anesthetized, and the abdomen hair was removed using depilatory cream (Veet, London, UK). After 24 hours for SC recovery, a container was fixed on the abdominal area free of hair with superglue in order to administer liquid sample without leakage. In all, $500 \mu \mathrm{L}$ of C6-loaded NPs with and without NIC, C6-NIC complex suspension, and aqueous suspension with an equal amount of C6 $(0.1 \%, \mathrm{w} / \mathrm{v})$ were added to the container, respectively. 
After 4 hours of administration, the formulation remaining on the skin was carefully removed. The skin was separated and sliced into sections of $0.2 \mathrm{~cm} \times 0.5 \mathrm{~cm}$ with a cryostat microtome (CM1900; Leica Microsystems, Wetzlar, Germany), and the skin slice was observed using CLSM.

\section{Cellular uptake of C6-loaded NPs}

HaCaT cell was obtained from China Center for Type Culture Collection (Wuhan, People's Republic of China). The cells were cultured in DMEM supplemented with FBS (10\%, v/v), $100 \mathrm{IU} / \mathrm{mL}$ penicillin, and $100 \mu \mathrm{g} / \mathrm{mL}$ streptomycin in the humidified atmosphere of $95 \%$ with $5 \% \mathrm{CO}_{2}$ at $37^{\circ} \mathrm{C}$. The medium was replenished every other day. The $\mathrm{HaCaT}$ cells were then cultured in a $15 \mathrm{~mm}^{2}$ confocal dish $\left(1 \times 10^{5}\right.$ cells/ well) with DMEM medium. After cultured for 24 hours, the culture medium was removed and washed with phosphate buffer solution for three times. In all, $200 \mu \mathrm{L}$ of HA-CholNPs-NIC, HA-Chol-NPs, NIC complex suspension, and aqueous suspension containing an equal amount of C6 $(0.1 \%$, w/v) were diluted by DMEM without FBS to $10 \mu \mathrm{g} / \mathrm{mL}$ and added to the dish, respectively. After culturing for 4 hours, the culture medium was removed and the cells were washed three times with phosphate buffer solution. Then the cells were fixed with $4 \%$ paraformaldehyde solution at ambient temperature for 15 minutes, and the nuclei of cells were labeled by DAPI. The cell uptake was evaluated using CLSM. The excitation and emission wavelengths of C6 were $430 \mathrm{~nm}$ and $485 \mathrm{~nm}$, respectively.

\section{HPLC analysis of FK506}

FK506 in different samples was determined using HPLC. HPLC system (Agilent 1260 series; Agilent Technologies, Santa Clara, CA, USA) consisted of quaternary pump (G1310A), degasser (G1322A), autosampler (G1329), column thermostat (CO-1000), UV detector (G1314A), and data processing software (Agilent ChemStation for LC systems; Agilent Technologies). A C8 column (Grace ${ }^{\circledR}$, Grace Davison Discovery Sciences, Deerfield, IL, USA, 250×4.6 mm, $5 \mu \mathrm{m})$ was used for FK506 analysis with water:isopropyl alcohol:tetrahydrofuran $(5: 2: 2, \mathrm{v} / \mathrm{v} / \mathrm{v})$ as the mobile phase at a flow rate of $0.8 \mathrm{~mL} / \mathrm{min}$ at $55^{\circ} \mathrm{C}$, and the detection wavelength was set at $220 \mathrm{~nm}$.

\section{Data analysis}

Each experiment was performed at least in triplicate. The data were given as mean \pm standard deviation (SD). The statistical differences between the groups used one-way analysis of variance followed by least significant difference as post hoc analysis (SPSS Version 19.0; IBM Corporation, Armonk, NY, USA). $P<0.05$ was considered statistically significant.

\section{Results and discussion Solubility}

NIC, vitamin $\mathrm{B}_{3}$, is a hydrophilic molecule and well known as a hydrotropic agent for its ability to enhance the solubility of poorly water-soluble drugs. ${ }^{37,38}$ As shown in Table 1, FK506 was a poorly water-soluble drug with the solubility of $0.69 \pm 0.24 \mu \mathrm{g} / \mathrm{mL}$, and the solubility of FK506 increased with the increase in NIC concentration. The solubility of FK506 in 30\% (w/v) NIC solution was 532-fold as that in water. Similar results of other poorly water-soluble drugs were reported by Sanghvi et $\mathrm{al}^{39}$ and Suzuki and Sunada. ${ }^{40}$ The mechanism of how NIC increased the solubility of poorly water-soluble drugs is still debated. The commonly proposed mechanisms were: i) the complex of NIC-drug formed owing to the interaction between NIC and the poorly soluble molecules ${ }^{30}$ and ii) self-aggregation of NIC might help segregate the hydrophobic drug from water, and the total energy of the solution was reduced. ${ }^{41}$ However, the exact mechanism of hydrotropic solubilization of FK506 by NIC will be the subject of subsequent research.

\section{Percutaneous permeation of FK506 in NIC solution}

As NIC improved the solubility of FK506, it was supposed that NIC could enhance the percutaneous permeation of FK506. The cumulative amount of FK506 permeated, retention, and flux from in vitro permeation are listed in Table 2, and the cumulative permeated amount of FK506 through the skin is plotted as a function of time (Figure 2). Based on Table 2, the cumulative permeated amount, retention, and flux of FK506 were enhanced in comparison with FK506 suspension when $10 \%(\mathrm{w} / \mathrm{v})$ or $20 \%(\mathrm{w} / \mathrm{v})$ NIC was used as the hydrotropic agent $(P<0.05)$. It was possibly attributed to the increased solubility of FK506 in NIC solution, which increased FK506 concentration gradient and partition of skin/ vehicle. Moreover, NIC could easily permeate into skin when 20\% NIC solution without FK506 was performed in in vitro

Table I Solubility of FK506 in different concentrations of NIC solution

\begin{tabular}{lllll}
\hline Solubility & Water & $\begin{array}{l}10 \%(w / v) \\
\text { NIC }\end{array}$ & $\begin{array}{l}20 \%(w / v) \\
\text { NIC }\end{array}$ & $\begin{array}{l}30 \%(w / v) \\
\text { NIC }\end{array}$ \\
\hline Solubility $(\mu g / \mathrm{mL})$ & $0.69 \pm 0.24$ & $39.29 \pm 1.86$ & $158.90 \pm 8.89$ & $367.63 \pm 12.82$ \\
\hline
\end{tabular}

Note: Data represent mean $\pm S D, n=4$ per group.

Abbreviations: FK506, tacrolimus; NIC, nicotinamide; SD, standard deviation. 
Table 2 Permeated amount, deposition, and flux $\left(U_{s s}\right)$ of FK506 after $24 \mathrm{~h}$ of permeation

\begin{tabular}{llll}
\hline $\begin{array}{l}\text { FK506 } \\
\text { sample }\end{array}$ & $\begin{array}{l}\text { Permeated } \\
\text { amount }\left(\mu \mathrm{g} / \mathrm{cm}^{2}\right)\end{array}$ & $\begin{array}{l}\text { Deposition } \\
\text { amount }\left(\mu \mathrm{g} / \mathrm{cm}^{2}\right)\end{array}$ & Flux $\left(\mu \mathrm{g} / \mathrm{cm}^{2} / \mathbf{h}\right)$ \\
\hline $\begin{array}{l}10 \% \mathrm{NIC} \\
(\mathrm{w} / \mathrm{v})\end{array}$ & $5.37 \pm 1.04^{*}$ & $1.37 \pm 0.33^{*}$ & $0.37 \pm 0.05^{*}$ \\
$\begin{array}{l}20 \% \mathrm{NIC} \\
(\mathrm{w} / \mathrm{v})\end{array}$ & $6.60 \pm 2.27^{*}$ & $1.71 \pm 0.32^{*}$ & $0.46 \pm 0.10^{*}$ \\
$30 \% \mathrm{NIC}$ & $4.35 \pm 1.30$ & $1.26 \pm 0.18$ & $0.25 \pm 0.09$ \\
$(\mathrm{w} / \mathrm{v})$ & & & \\
Suspension & $2.33 \pm 1.26$ & $0.54 \pm 0.09$ & $0.20 \pm 0.10$ \\
\hline
\end{tabular}

Notes: $* P<0.05$ in comparison with FK506 aqueous suspension. Data represent mean $\pm S D, n=4$ per group.

Abbreviations: FK506, tacrolimus; h, hours; NIC, nicotinamide; SD, standard deviation.

permeation studies (data not shown). When the complex of FK506-NIC formed or self-aggregated NIC segregated FK506 from water in the NIC hydrotropic system, FK506 permeation might have enhanced due to the driving force from NIC permeation. Interestingly, when FK506 was present in $20 \%$ NIC solution, the cumulative permeated amount, retention, and flux were higher than that in $10 \%$ NIC solution or $30 \%$ NIC solution. Therefore, $20 \%$ NIC solution was chosen as the hydrotropic solution for preparing NPs.

\section{Preparation and characterization of NPs}

HA, a hydrophilic component of skin and human body, possesses several properties, including viscoelasticity, biocompatibility, hydration, and lubrication. Chol is also a hydrophobic component of human skin. Amphiphilic HA

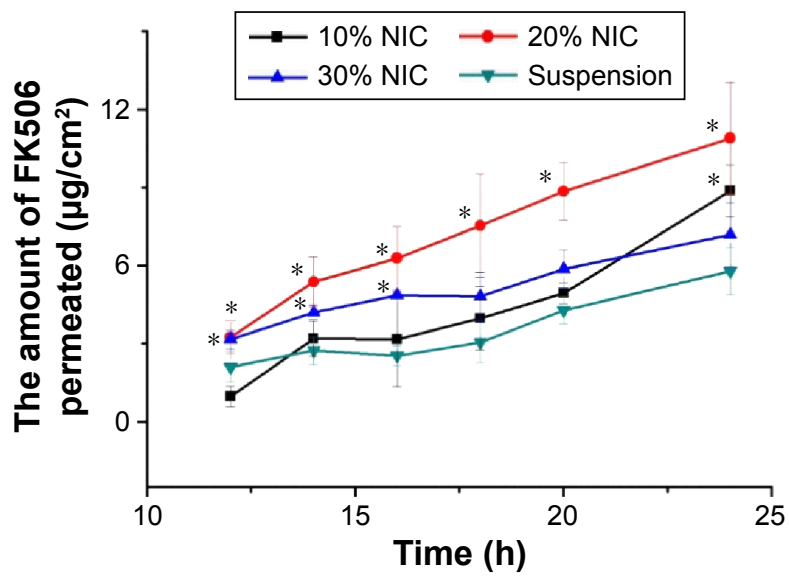

Figure 2 In vitro skin percutaneous studies of FK506 from different concentrations of NIC solution.

Notes: The percutaneous studies were performed with vertical Franz diffusion cell at $32^{\circ} \mathrm{C} \pm 1{ }^{\circ} \mathrm{C}$. Data represent mean $\pm \mathrm{SD}, \mathrm{n}=4$ per group. $* \mathrm{P}<0.05$ in comparison with FK506 aqueous suspension. The concentration of FK506 in each formulation was $1 \mathrm{mg} / \mathrm{mL}$.

Abbreviations: FK506, tacrolimus; h, hours; NIC, nicotinamide; $10 \%$ NIC, FK506 dissolved in 10\% nicotinamide aqueous solution; $20 \%$ NIC, FK506 dissolved in $20 \%$ nicotinamide aqueous solution; 30\% NIC, FK506 dissolved in 30\% nicotinamide aqueous solution; SD, standard deviation. derivatives were synthesized by conjugating Chol with HA to form HA-Chol, which could self-assemble to be NPs following incorporation of hydrophobic drugs into the hydrophobic core of NPs. In the present study, the preparation of HA-Chol conjugates was according to the previous report (Figure 1A). ${ }^{24}$ Chol including a linker, ethanediamine, was attached. Then the $-\mathrm{NH}_{2}$ of Chol-Am could conjugate with the $-\mathrm{COOH}$ of $\mathrm{HA}$ by amide bond formation. By increasing the feed of Chol, HA-Chol with different DSs was obtained (Table 3). The ${ }^{1} \mathrm{H}$ NMR spectrum of HA-Chol is presented in Figure 1B. The methine group of Chol $(\delta=5.32 \mathrm{ppm}$ $[1 \mathrm{H},-\mathrm{CH}])$ and the $N$-acetyl group in the sugar unit of HA $(\delta=2.10 \mathrm{ppm}[3 \mathrm{H},-\mathrm{COCH} 3])$ were confirmed in the ${ }^{1} \mathrm{H}$ NMR spectrum of HA-Chol, indicating successful synthesis of HA-Chol.

The CAC of HA-Chol was determined using the fluorescence excitation spectra method. Figure 3 shows the plot of fluorescence intensity ratio $\left(I_{388} / I_{375}\right)$ to the logarithmic of concentration of HA-Chol conjugates. The CAC was determined by the HA-Chol threshold concentration to form self-assembled NPs. As shown in Figure 3, the CAC decreased with the increase in Chol DS. It was $43.7 \mu \mathrm{g} / \mathrm{mL}$ for HA-Chol 1, $11.5 \mu \mathrm{g} / \mathrm{mL}$ for HA-Chol 2, and $2.04 \mu \mathrm{g} / \mathrm{mL}$ for HA-Chol 3. Based on these data, HA-Chol could selfassemble to be NPs to incorporate poorly water-soluble drugs into their hydrophobic inner core.

NPs were prepared by the sonication method. The morphology of NPs characterized by AFM was uniformly spherical (Figure 4). The particle size and zeta potential of HA-Chol-NPs with different DSs were examined by dynamic light scattering, and the results are shown in Table 3. The average size of HA-Chol-NPs in the absence of NIC was larger than that in the presence of NIC, and the size of HA-Chol-NPs with NIC ranged from $160.5 \mathrm{~nm}$ to $208.0 \mathrm{~nm}$, and the zeta potential ranged from $-31.5 \mathrm{mV}$ to $-41.9 \mathrm{mV}$.

Table 3 Characteristics of HA-Chol-NPs

\begin{tabular}{llllll}
\hline Sample & DS & Size $(\mathrm{nm})$ & PDI & $\xi(\mathrm{mV})$ & EE $(\%)$ \\
\hline HA-Chol-NPs I & 0.39 & $243.2 \pm 14.2$ & $0.263 \pm 0.047$ & $-43.3 \pm 3.4$ & $72.1 \pm 3.0$ \\
HA-Chol-NPs- & 0.39 & $208.0 \pm 2.2$ & $0.224 \pm 0.031$ & $-41.9 \pm 3.0$ & $79.2 \pm 7.2$ \\
NIC I & & & & & \\
HA-Chol-NPs- & 0.47 & $181.4 \pm 6.8$ & $0.200 \pm 0.027$ & $-40.5 \pm 7.0$ & $77.9 \pm 9.6$ \\
NIC 2 & & & & & \\
HA-Chol-NPs- & 0.81 & $160.5 \pm 4.7$ & $0.189 \pm 0.014$ & $-31.5 \pm 1.4$ & $79.3 \pm 0.6$ \\
NIC 3 & & & & & \\
\hline
\end{tabular}

Note: Data represent mean $\pm S D, n=4$ per group.

Abbreviations: DS, degree of substitution; $E E$, encapsulation efficiency; HA-Chol-NPs, hyaluronic acid-cholesterol nanoparticles; HA-Chol-NPsNIC, hyaluronic acid-cholesterol nanoparticles containing nicotinamide; PDI, polydispersity index; $\xi$, zeta potential; SD, standard deviation. 


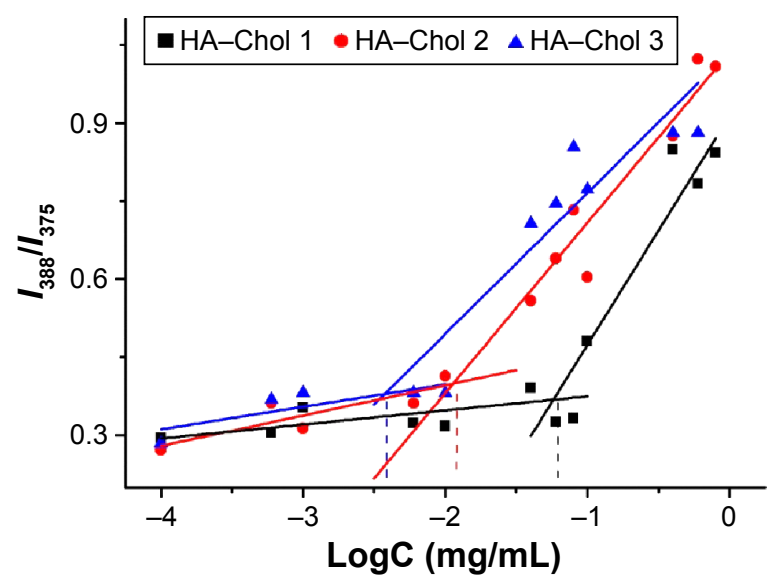

Figure 3 The plot of fluorescence intensity ratio $\left(I_{388} / I_{375}\right)$ to the logarithmic of concentration (LogC) of HA-Chol conjugates with different DSs.

Note: The CAC of HA-Chol with different DSs was the concentration under the inflection point of the curve.

Abbreviations: CAC, critical aggregation concentration; DS, degree of substitution; HA-Chol, hyaluronic acid-cholesterol conjugates.

With the increase in Chol DS, the particle size was decreased due to the increasing hydrophobic interaction. The negative zeta potential was attributed to the negative charge of the carboxylic group of HA backbone. The high zeta potential might prevent the aggregation of NPs in solution by electrostatic repulsion.

The EE was 79.2\% $\% 7.2 \%, 77.9 \% \pm 9.2 \%$, and $79.3 \% \pm 0.6 \%$ for HA-Chol 1, HA-Chol 2, and HA-Chol 3, respectively. The EE was not influenced by the DS of Chol, although the latter could increase the lipophilic core of NPs. A similar result was also obtained by another group. ${ }^{42}$ Comparing

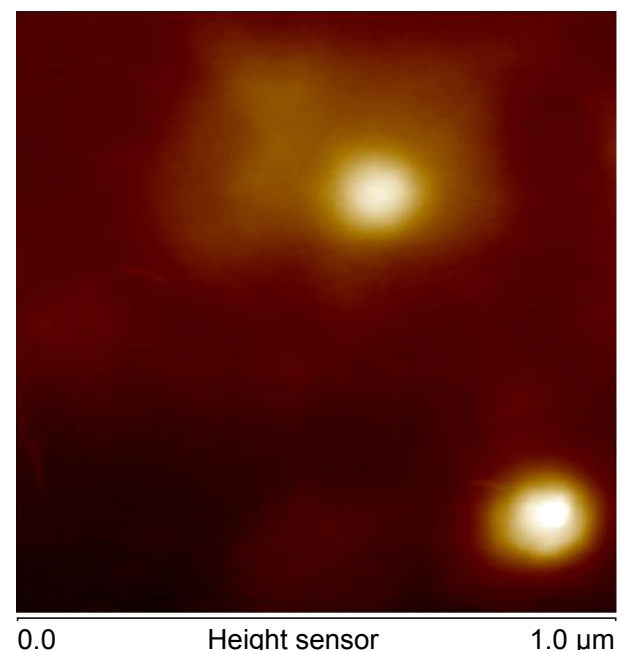

$-571.9 \mathrm{~nm}$

Figure 4 The morphology of FK506-HA-Chol-NPs-NIC observed by AFM at room temperature.

Abbreviations: AFM, atomic force microscope; FK506-HA-Chol-NPs-NIC, tacrolimus-loaded hyaluronic acid-cholesterol nanoparticles containing nicotinamide. the EE of HA-Chol 1 with and without NIC, the EE of HA-Chol 1 with NIC was higher than that of without NIC $(72.1 \% \pm 3.0 \%)$. The result suggested that NIC could enhance the drug incorporation of NPs through increasing the solubility and decreasing the high lipophilicity of FK506. In order to screen the HA-Chol, in vitro permeation of FK506 from HA-Chol-NPs-NIC with different HA-Chol DSs has been carried out. The permeated amount of HA-Chol 1-NPsNIC after 24 hours of permeation was $13.38 \pm 2.26 \mu \mathrm{g} / \mathrm{cm}^{2}$, which was significantly higher than that of HA-Chol 2-NPs-NIC $\left(6.86 \pm 1.69 \mu \mathrm{g} / \mathrm{cm}^{2}, P<0.05\right)$ or HA-Chol $3-N P s-N I C\left(4.74 \pm 0.91 \mu \mathrm{g} / \mathrm{cm}^{2}, P<0.05\right)$. The deposition and flux of FK506 from HA-Chol 1-NPs-NIC were $2.39 \pm 0.53 \mu \mathrm{g} / \mathrm{cm}^{2}$ and $0.86 \pm 0.27 \mu \mathrm{g} / \mathrm{cm}^{2} / \mathrm{h}$, respectively, which were significantly higher than those from HA-Chol 2-NPs-NIC or HA-Chol 3-NPs-NIC $\left(1.22 \pm 0.19 \mu \mathrm{g} / \mathrm{cm}^{2}\right.$, $P<0.05$, and $0.44 \pm 0.15 \mu \mathrm{g} / \mathrm{cm}^{2} / \mathrm{h}, P<0.05$, respectively, for HA-Chol 2-NPs-NIC and $1.14 \pm 0.40 \mu \mathrm{g} / \mathrm{cm}^{2}, P<0.05$, and $0.31 \pm 0.09 \mu \mathrm{g} / \mathrm{cm}^{2} / \mathrm{h}, P<0.05$, respectively, for HA-Chol 3-NPs-NIC). Based on these results, HA-Chol 1 possessing low DS, high EE, and high permeability was used for the following experiments.

\section{In vitro permeation of FK506-NPs}

As reported in previous studies, the application of NPs as the vehicle for percutaneous drug delivery achieved more retention of drugs in the skin for prolonging the residence time and targeting upper layer of skin. ${ }^{43,44}$ In the present study, we used HA-Chol-NPs as the carrier and NIC as the hydrotrope to prepare FK506-HA-Chol-NPs-NIC system and evaluate their effects on FK506 permeation into and across the skin. The cumulative permeated amount of FK506 was plotted versus time, and the retention of FK506 in the skin is shown in Figure 5 and Table 4. The commercial preparation (Protopic, 0.1\%,w/v) and 0.1\% (w/v) FK506 aqueous suspension were taken as the reference group and control group, respectively. As shown in Figure 5 and Table 4, the FK506 permeation from the NIC complex or HA-CholNPs was higher than that from Protopic or suspension after 24 hours of permeation, and there was no significant difference between Protopic and suspension $(P>0.05)$. The cumulative permeated amount of FK506 from NPs or NIC complex was, respectively, 1.53-fold or 1.70-fold as that of Protopic, and the skin retention of FK506 from NPs or NIC complex was, respectively, 2.05-fold or 4.17-fold as that of Protopic. What is more, FK506 from NPs-NIC showed the highest permeation at all time points as compared with NPs or the NIC complex group. The cumulative permeated 


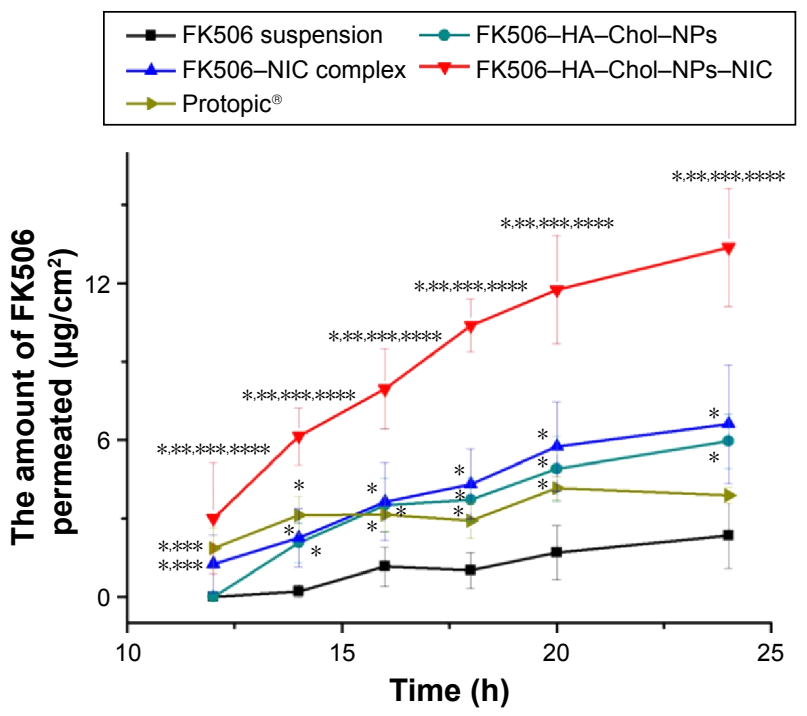

Figure 5 In vitro skin percutaneous studies of FK506 from different vehicles. Notes: Data represent mean $\pm S D, n=4$ per group. *Significantly different $(P<0.05)$ in comparison with FK506 aqueous suspension. **Significantly different $(P<0.05)$ in comparison with Protopic, $0.1 \%$, w/w. $* * *$ Significantly different $(P<0.05)$ in comparison with FK506-NPs. ****Significantly different $(P<0.05)$ in comparison with FK506-NIC solution. The concentration of FK506 in each formulation was I $\mathrm{mg} / \mathrm{mL}$.

Abbreviations: FK506, tacrolimus; FK506-HA-Chol-NPs, tacrolimus-loaded hyaluronic acid-cholesterol nanoparticles; FK506-HA-Chol-NPs-NIC, tacrolimusloaded hyaluronic acid-cholesterol nanoparticles containing nicotinamide; FK506-NIC complex, tacrolimus-nicotinamide complex; FK506-NPs, tacrolimusnanoparticles complex; h, hours; SD, standard deviation.

amount of FK506 was $13.38 \pm 2.26 \mu \mathrm{g} / \mathrm{cm}^{2}$ after 24 hours of permeation, which was significantly higher than that of the NIC complex, $6.60 \pm 2.27 \mu \mathrm{g} / \mathrm{cm}^{2}(P<0.05)$, or NPs alone, $5.96 \pm 1.05 \mu \mathrm{g} / \mathrm{cm}^{2}(P<0.05)$, and the FK506 retention in the skin from NPs-NIC presented the highest retention $\left(2.39 \pm 0.53 \mu \mathrm{g} / \mathrm{cm}^{2}\right)$ than NPs $\left(0.84 \pm 0.14 \mu \mathrm{g} / \mathrm{cm}^{2}\right)$ or the NIC complex $\left(1.71 \pm 0.32 \mu \mathrm{g} / \mathrm{cm}^{2} ; P<0.05\right)$. The results indicated that either NPs carrier or NIC hydrotropy could enhance the permeation of FK506, and the combination of HA-Chol-NPs with NIC could synergistically enhance the permeation of FK506 into and through the skin.

There were various mechanisms to elucidate how NPs enhanced drug penetration through the skin. First, NPs were used as drug carriers and accumulated on the skin surface to create high local concentration of loaded drugs, which facilitated the drug-sustained release and permeation in the skin. ${ }^{45,46}$ Second, NPs were reported to penetrate into the skin through skin appendages such as hair follicles. ${ }^{47}$ Hair follicles occupied $\sim 0.1 \%$ of the entire skin. ${ }^{48} \mathrm{NPs}$ transported into the hair follicles, resulting in enhanced penetration of drugs loaded by NPs. In this project, both the penetration and retention of FK506 from HA-Chol-NPs were enhanced, which indicated that HA-Chol-NPs possibly played the role of drug carrier and also penetrated through the skin appendages. For FK506-NIC, as discussed above, the penetration and retention enhancement were possibly ascribed to: i) the increased solubility of FK506 in NIC solution, which increased FK506 concentration gradient and partition of skin/ vehicle, and ii) when the complex of FK506-NIC formed or self-aggregated NIC segregated FK506 from water in the NIC hydrotropic system, FK506 permeation might have enhanced due to the driving force from NIC permeation. The reasons that HA-Chol-NPs combined with NIC facilitated percutaneous drug delivery could be attributed to solubilizing, nanoloading, and partitioning effects. The results indicated that the combination of HA-Chol-NPs with NIC was a promising approach for FK506 percutaneous delivery system.

If there is more amount of percutaneous drug delivery, skin diseases are treated more effectively, which has been backed by many reports. ${ }^{8,49,50}$ As we described earlier, FK506-loaded ethosomes, lipid NPs, or modified lipid NPs were reported to enhance FK506 penetration, resulting in an enhanced efficacy for treating AD. ${ }^{8,49,50}$ Therefore, the synergistically enhanced penetration of FK506 by the combination of HA-Chol-NPs with NIC also suggests that FK506-HA-Chol-NPs-NIC will exert an enhanced effect on treating skin diseases compared to FK506-HA-CholNPs, FK506-NIC complex, FK506 aqueous suspension, or commercial ointment. In clinic, FK506 has been investigated successfully in the management of $\mathrm{AD}$ and psoriasis. The

Table 4 Permeated amount, deposition, and flux $\left(U_{s s}\right)$ of FK506 after $24 \mathrm{~h}$ of permeation

\begin{tabular}{|c|c|c|c|}
\hline Formulation & Permeated amount $\left(\mu \mathrm{g} / \mathrm{cm}^{2}\right)$ & Deposition amount $\left(\mu \mathrm{g} / \mathrm{cm}^{2}\right)$ & Flux $\left(\mu \mathrm{g} / \mathrm{cm}^{2} / \mathrm{h}\right)$ \\
\hline HA-Chol-NPs & $5.96 \pm 1.05^{*}$ & $0.84 \pm 0.14$ & $0.47 \pm 0.08 * * * *$ \\
\hline FK506-NIC complex & $6.60 \pm 2.27 * * *$ & $1.71 \pm 0.32^{* * * * * * * *}$ & $0.46 \pm 0.10^{* * * *}$ \\
\hline HA-Chol-NPs-NIC & $13.38 \pm 2.26 * * * * * * *, * * * * *$ & $2.39 \pm 0.53 *, * *, * * *, * * * *$ & $0.86 \pm 0.27 *, * *, * * *, * * * *$ \\
\hline Suspension & $2.33 \pm 1.26$ & $0.54 \pm 0.09$ & $0.20 \pm 0.10$ \\
\hline Protopic & $3.89 \pm 0.30$ & $0.4 I \pm 0.20$ & $0.16 \pm 0.05$ \\
\hline
\end{tabular}

Notes: *Significantly different $(P<0.05)$ compared to FK506 aqueous suspension. **Significantly different $(P<0.05)$ compared to Protopic, $0.1 \%$, w/w. ***Significantly different $(P<0.05)$ compared to FK506 NPs. ****Significantly different $(P<0.05)$ compared to FK506-NIC solution. Data represent mean \pm SD, $n=4$ per group.

Abbreviations: FK506, tacrolimus; FK506-NIC complex, tacrolimus-nicotinamide complex; h, hours; HA-Chol-NPs, hyaluronic acid-cholesterol nanoparticles; HA-CholNPs-NIC, hyaluronic acid-cholesterol nanoparticles containing nicotinamide; SD, standard deviation. 
formulation of FK506-HA-Chol-NPs-NIC exhibits attractive features for FK506 clinical setting as follows. First, the enhanced percutaneous penetration of FK 506 by the delivery system as mentioned earlier facilitated the topical efficacy for these diseases. Second, the materials to construct HA-CholNPs-NIC, especially HA and NIC, also provided advantages for percutaneous delivery. Generally, HA was thought to act as a mucoadhesive and retain the drug at its site of action. When applied topically, HA presented localizing delivery of drug to the epidermis, ${ }^{17}$ thus obtaining a targeting therapeutic treatment effect for $\mathrm{AD}$ and psoriasis. NIC has been reported as a potential addition of anti-AD armamentarium, ${ }^{32}$ and it is a cheap generic vehicle with a well-established safety profile in human beings.

\section{Percutaneous delivery of C6-loaded NPs in vivo}

The deposition of fluorescently labeled formulations was evaluated using CLSM. C6 was employed as a hydrophobic fluorescent probe. The skin permeation from $\mathrm{C} 6$ aqueous suspension, 20\% (w/v) NIC solution, C6-loaded HA-Chol-NPs, and C6-loaded HA-Chol-NPs-NIC was observed. The results of CLSM experiments following 4 hours of incubation are illustrated in Figure 6. After 4 hours of application, the confocal images presented that the aqueous suspension delivered very little C6 into the skin (Figure 6A). When C6 was hydrotropically solubilized in $20 \%$ NIC solution, $\mathrm{C} 6$ could permeate into deeper skin layers (Figure 6B). However, when C6 was loaded in HA-Chol-NPs, the strong fluorescence intensity was only distributed in SC and hair follicles (Figure 6C), which indicated that NPs played predominantly the role of drug carrier after 4 hours of permeation. The fluorescence intensity of C6-loaded HA-Chol-NPs or C6-NIC solution was stronger than that of C6 suspension, indicating that either NIC or HA-Chol-NPs alone could enhance the retention of drugs in the skin. When C6 was loaded in the HA-Chol-NPs-NIC system, the fluorescence intensity within the skin was significantly higher than that of the NIC solution or HA-Chol-NPs alone, especially in the epidermis (Figure 6D). These results supported the results from the in vitro permeation experiments and confirmed the assumption that HA-Chol-NPs-NIC presented a synergistic effect on drug permeation in the skin after the drug incorporated into NPs with a hydrotropic NIC system.

\section{Cell uptake of C6-loaded NPs into $\mathrm{HaCaT}$}

$\mathrm{SC}$ was the main barrier of skin. For the purpose of delivering therapeutic agents to target site of skin by NPs, one of the

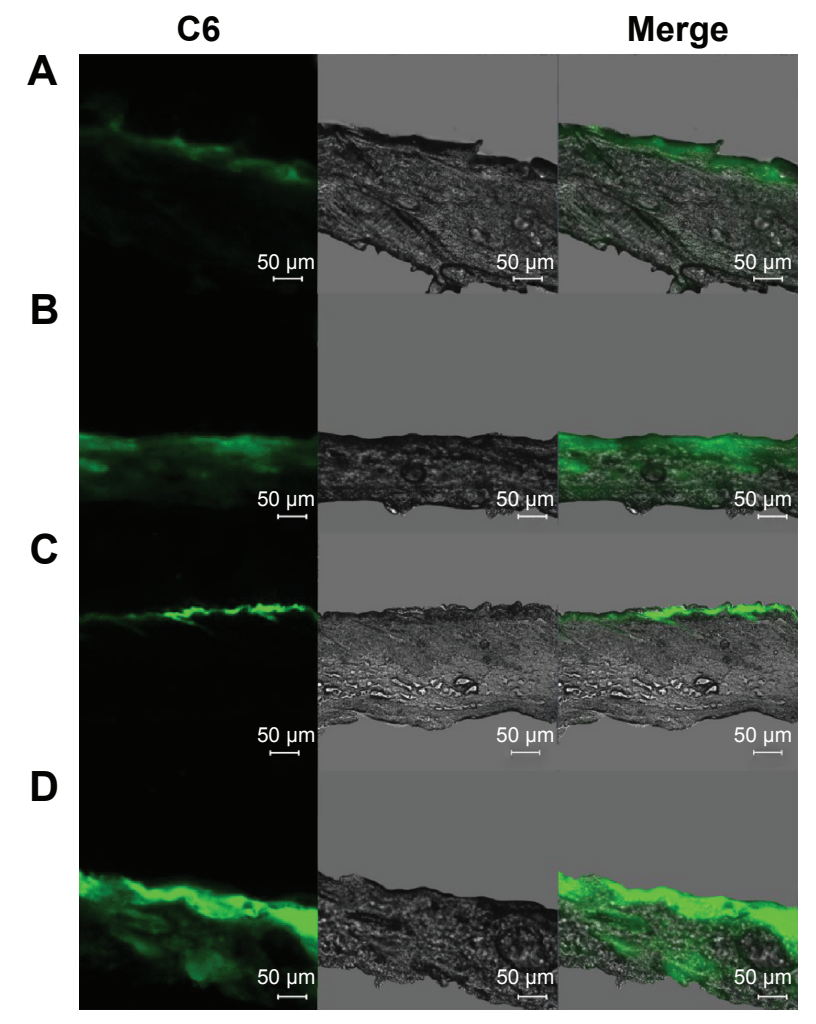

Figure 6 Confocal laser scanning microscopy of skin sections of Sprague Dawley rats after 4 hours of in vivo administration of (A) C6 aqueous suspension, (B) C6-NIC complex suspension, (C) C6-HA-Chol-NP suspension, and (D) C6-HAChol-NPs-NIC suspension.

Notes: The concentration of $\mathrm{C} 6$ in each formulation was $1 \mathrm{mg} / \mathrm{mL}$, and the green fluorescence represented C6. Scale bar: $50 \mu \mathrm{m}$. Magnification $\times 100$.

Abbreviations: $\mathrm{C} 6$, coumarin 6; C6-HA-Chol-NPs, coumarin 6-loaded hyaluronic acid-cholesterol nanoparticles; C6-HA-Chol-NPs-NIC, coumarin 6-loaded hyaluronic acid-cholesterol nanoparticles containing nicotinamide; C6-NIC complex, coumarin 6-nicotinamide complex; h, hours.

possibilities was that NPs, as the carrier, could be actively internalized by keratinocytes or other cells of the epidermis. ${ }^{51}$ Immortalized $\mathrm{HaCaT}$, similar to normal keratinocytes, were chosen to evaluate the cellular uptake of HA-Chol-NPs. Cell uptake studies were carried out using C6 as fluorescence probe, which has been widely used in cell uptake experiments for observation by CLSM. ${ }^{52-54}$ The uptake of C6-loaded HA-Chol-NPs-NIC, C6-loaded HA-Chol-NPs, C6 in 20\% (w/v) NIC solution, and C6 aqueous suspension containing an equal amount of $\mathrm{C} 6(10 \mu \mathrm{g} / \mathrm{mL})$ by HaCaT cell was evaluated. As shown in Figure 7A, the cell showed no background fluorescence interference except the nucleus labeled by DAPI in the DMEM group. After 4 hours of incubation, the $\mathrm{C} 6$ suspension group presented negligible fluorescence, indicating very little C6 uptake by HaCaT cells (Figure 7B). NIC solution could slightly enhance the cell uptake of C6 compared with that of C6 suspension (Figure 7C). However, when C6 was loaded in HA-Chol-NPs, its uptake by $\mathrm{HaCaT}$ cells was significantly enhanced (Figure 7D). It has 


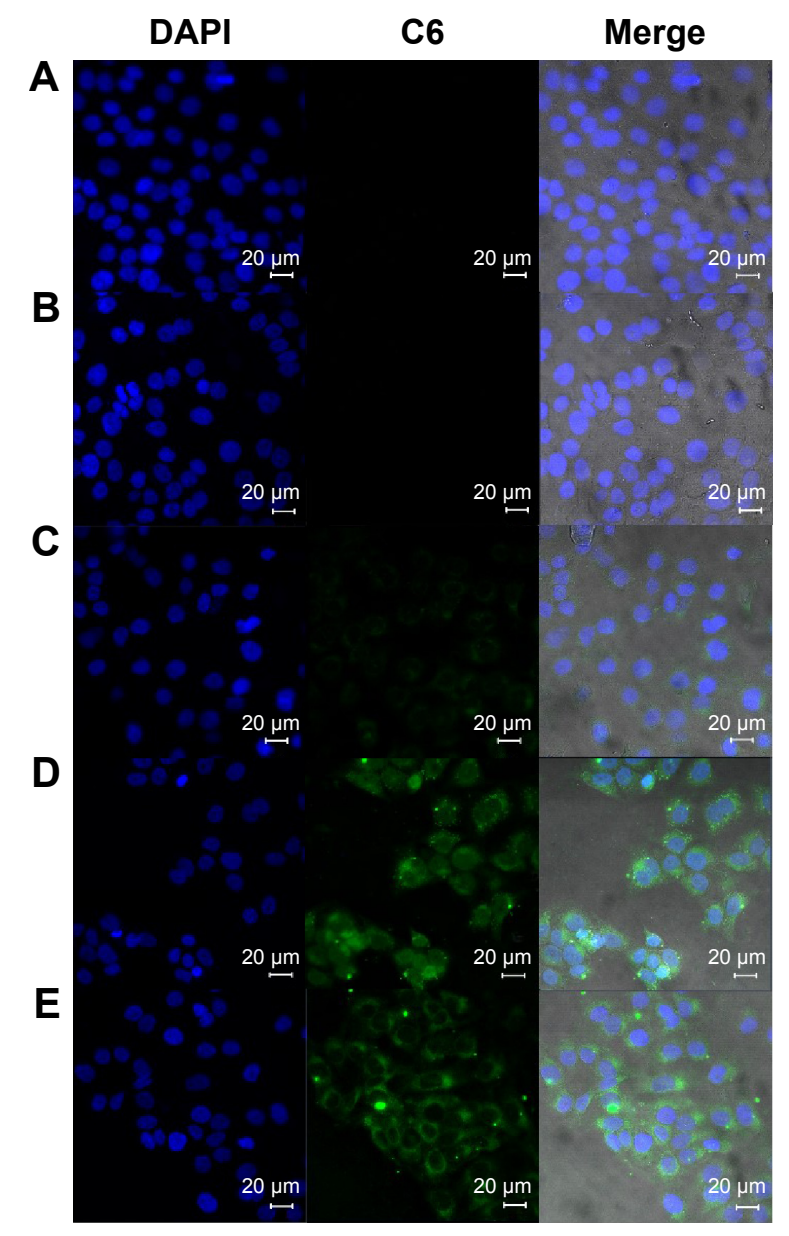

Figure 7 Confocal laser scanning microscopy of cell uptake of $\mathrm{HaCaT}$ cell after 4 hours of administration of (A) DMEM; (B) C6 aqueous suspension; (C) C6-NIC complex suspension; (D) C6-HA-Chol-NP suspension; and (E) C6-HA-CholNPs-NIC suspension.

Notes: The concentration of $\mathrm{C} 6$ in each formulation was $10 \mu \mathrm{g} / \mathrm{mL}$. Cells were stained for nuclei (in blue) and C6 (in green). Scale bar: $20 \mu \mathrm{m}$. Magnification $\times 400$. Abbreviations: $\mathrm{C} 6$, coumarin 6; C6-HA-Chol-NP, coumarin 6-loaded hyaluronic acid-cholesterol nanoparticle; C6-HA-Chol-NPs-NIC, coumarin 6-loaded hyaluronic acid-cholesterol nanoparticles containing nicotinamide; C6-NIC complex, coumarin 6-nicotinamide complex; DAPI, 4',6-diamidino-2-phenylindole; DMEM, Dulbecco's Modified Eagle's Medium; h, hours; $\mathrm{HaCaT}$, human keratinocytes.

been reported that CD44 expressed in $\mathrm{HaCaT}$ cells $^{55}$ and HA-Chol-NPs could specifically bind to CD44 receptor expressing on $\mathrm{HaCaT}$ cells and resulted in uptake by $\mathrm{HaCaT}$ cells through CD44 receptor-mediated endocytosis, which would facilitate the uptake of loaded drugs by cells. When C6 was loaded in HA-Chol-NPs with NIC, the cell uptake presented similar green fluorescence to that of HA-Chol-NPs without NIC (Figure 7E), which indicated that cell uptake was mainly ascribed to HA-Chol-NPs and NIC would not hinder the uptake.

\section{Conclusion}

Although topical administration of FK506 was effective in the treatment of various inflammatory skin diseases, the formulation for FK506 percutaneous delivery remained a challenge due to its high hydrophobicity and high MW. In this study, amphiphilic HA-Chol conjugates were synthesized. Hydrotropic NIC was used to solubilize FK506 and facilitate FK506 to be loaded into HA-Chol self-assembling NPs. In vitro permeation investigation showed that either NIC or HA-Chol-NPs alone significantly enhanced FK506 permeation through and into the skin, and the combination of HA-Chol-NPs with NIC exhibited a significantly synergistic effect on FK506 permeation through and into the skin in comparison with NIC or HA-Chol-NPs alone. The CLSM study further confirmed the synergistic percutaneous delivery effect of HA-Chol-NPs-NIC, and HaCaT cell uptake identified the enhancement of active agents delivered into the cells by HA-Chol-NPs. Overall, the results indicate that the combination of HA-Chol-NPs with NIC can exploit the advantages of both HA-Chol-NPs and NIC to enhance the solubility and permeability of FK506, which supports that the hybrid system of HA-Chol-NPs combined with NIC may be a promising approach for FK506 to effectively treat skin diseases. Nevertheless, further works such as the interaction of HA-Chol-NPs with NIC, in vivo penetration, and pharmacodynamic studies need to be carried out in order to obtain more information on HA-Chol-NPs-NIC as a percutaneous delivery system for FK506.

\section{Acknowledgment}

The work was financially supported by the National Natural Science Foundation of China (No 81073066 and No 81473358).

\section{Disclosure}

The authors report no conflicts of interest in this work.

\section{References}

1. Honbo T, Kobayashi M, Hane K, Hata T, Ueda Y. The oral dosage form of FK-506. Transplant Proc. 1987;19(5 suppl 6):S17-S22.

2. Hanifin JM, Ling MR, Langley R, Brenema D, Rafal E. Tacrolimus ointment for the treatment of atopic dermatitis in adult patients: part I, efficacy. J Am Acad Dermatol. 2001;44(1 suppl):S28-S38.

3. Wollenberg A, Reitamo S, Atzori F, et al. Proactive treatment of atopic dermatitis in adults with $0.1 \%$ tacrolimus ointment. Allergy. 2008;63(6) $742-750$.

4. Ezquerra GM, Regana MS, Acosta EH, Millet PU. Topical tacrolimus for the treatment of psoriasis on the face, genitalia, intertriginous areas and corporal plaques. J Drugs Dermatol. 2006;5(4):334-336.

5. Lauerma AI, Stein BD, Homey B, Lee CH, Bloom E, Maibach HI. Topical FK506: suppression of allergic and irritant contact dermatitis in the guinea pig. Arch Dermatol Res. 1994;286(6):337-340.

6. Goebel AS, Neubert RH, Wohlrab J. Dermal targeting of tacrolimus using colloidal carrier systems. Int J Pharm. 2011;404(1-2):159-168.

7. Yamanaka M, Yokota S, Iwao Y, Noguchi S, Itai S. Development and evaluation of a tacrolimus cream formulation using a binary solvent system. Int J Pharm. 2014;464(1-2):19-26. 
8. Li G, Fan Y, Fan C, et al. Tacrolimus-loaded ethosomes: physicochemical characterization and in vivo evaluation. Eur J Pharm Biopharm. 2012; 82(1):49-57.

9. Pople PV, Singh KK. Targeting tacrolimus to deeper layers of skin with improved safety for treatment of atopic dermatitis. Int J Pharm. 2010;398(1-2):165-178.

10. Pople PV, Singh KK. Development and evaluation of colloidal modified nanolipid carrier: application to topical delivery of tacrolimus. Eur $J$ Pharm Biopharm. 2011;79(1):82-94.

11. Gomes MJ, Martins S, Ferreira D, Segundo MA, Resis S. Lipid nanoparticles for topical and transdermal application for alopecia treatment: development, physicochemical characterization, and in vitro release and penetration studies. Int J Nanomedicine. 2014;9:1231-1242.

12. Filon FL, Mauro M, Adami G, Bovenzi M, Crosera M. Nanoparticles skin absorption: new aspects for a safety profile evaluation. Regul Toxicol Pharmacol. 2015;72(2):310-322.

13. Try C, Moulari B, Béduneau A, et al. Size dependent skin penetration of nanoparticles in murine and porcine dermatitis models. Eur J Pharm Biopharm. 2016;100:101-108.

14. Weissmann B, Meyer K. The structure of hyalobiuronic acid and of hyaluronic acid from umbilical Cord1, 2. J Am Chem Soc. 1954;76(7): 1753-1757.

15. Balazs EA. Therapeutic use of hyaluronan. Struct Chem. 2009; 20(2): 341-349.

16. Gaffney J, Matou-Nasri S, Grau-Olivares M, Slevin M. Therapeutic applications of hyaluronan. Mol Biosyst. 2010;6(3):437-443.

17. Brown MB, Jones SA. Hyaluronic acid: a unique topical vehicle for the localized delivery of drugs to the skin. J Eur Acad Dermatol Venereol. 2005;19(3):308-318.

18. Moore AR, Willoughby DA. Hyaluronan as a drug delivery system for diclofenac: a hypothesis for mode of action. Int J Tissue React. 1995; 17(4):153-156.

19. Brown TJ, Alcorn D, Fraser JR. Absorption of hyaluronan applied to the surface of intact skin. J Invest Dermatol. 1999;113(5):740-746.

20. Wang C, Tammi M, Tammi R. Distribution of hyaluronan and its CD44 receptor in the epithelia of human skin appendages. Histochemistry. 1992;98(2):105-112.

21. Tammi R, Säämänen AM, Maibach HI, Tammi M. Degradation of newly synthesized high molecular mass hyaluronan in the epidermal and dermal compartments of human skin in organ culture. J Invest Dermatol. 1991;97(1):126-130.

22. Tripodo G, Trapani A, Torre ML, Giammona G, Trapani G, Mandracchia D. Hyaluronic acid and its derivatives in drug delivery and imaging: recent advances and challenges. Eur J Pharm Biopharm. 2015;97(pt B):400-416.

23. Choi KY, Chung H, Min KH, et al. Self-assembled hyaluronic acid nanoparticles for active tumor targeting. Biomaterials. 2010;31(1): 106-114.

24. Choi KM, Jang M, Kim JH, Ahn HJ. Tumor-specific delivery of siRNA using supramolecular assembly of hyaluronic acid nanoparticles and 2b RNA-binding protein/siRNA complexes. Biomaterials. 2014; 35(25):7121-7132.

25. Song S, Qi H, Xu J, et al. Hyaluronan-based nanocarriers with CD44-overexpressed cancer cell targeting. Pharm Res. 2014;31(11): 2988-3005

26. Koo H, Min KH, Lee SC, et al. Enhanced drug-loading and therapeutic efficacy of hydrotropic oligomer-conjugated glycol chitosan nanoparticles for tumor-targeted paclitaxel delivery. J Control Release. 2013; 172(3):823-831

27. Rasool AA, Hussain AA, Dittert LW. Solubility enhancement of some water-insoluble drugs in the presence of nicotinamide and related compounds. J Pharm Sci. 1991;80(4):387-393.

28. Lim LY, Go ML. Caffeine and nicotinamide enhances the aqueous solubility of the antimalarial agent halofantrine. Eur J Pharm Sci. 2000;10(1): 17-28.

29. Huang Y, Zhang B, Gao Y, Zhang J, Shi L. Baicalein-nicotinamide cocrystal with enhanced solubility, dissolution, and oral bioavailability. J Pharm Sci. 2014;103(8):2330-2337.
30. Fan X, Chen J, Shen Q. Docetaxel-nicotinamide complex-loaded nanostructured lipid carriers for transdermal delivery. Int J Pharm. 2013; 458(2):296-304.

31. Virág L, Szabó C. The therapeutic potential of poly(ADP-ribose) polymerase inhibitors. Pharmacol Rev. 2002;54(3):375-429.

32. Namazi MR. Nicotinamide as a potential addition to the anti-atopic dermatitis armamentarium. Int Immunopharmacol. 2004;4(6):709-712.

33. Niren NM. Pharmacologic doses of nicotinamide in the treatment of inflammatory skin conditions: a review. Cutis. 2006;77(1 suppl):11-16.

34. Levine D, Even-Chen Z, Lipets I, et al. Pilot, multicenter, doubleblind, randomized placebo-controlled bilateral comparative study of a combination of calcipotriene and nicotinamide for the treatment of psoriasis. J Am Acad Dermatol. 2010;63(5):775-781.

35. Bard B, Martel S, Carrupt PA. High throughput UV method for the estimation of thermodynamic solubility and the determination of the solubility in biorelevant media. Eur J Pharm Sci. 2008;33(3):230-240.

36. Che J, Wu Z, Shao W, et al. Synergetic skin targeting effect of hydroxypropyl- $\beta$-cyclodextrin combined with microemulsion for ketoconazole. Eur J Pharm Biopharm. 2015;93:136-148.

37. Nicoli S, Zani F, Bilzi S, Bettini R, Santi P. Association of nicotinamide with parabens: effect on solubility, partition and transdermal permeation. Eur J Pharm Biopharm. 2008;69(2):613-621.

38. Coffman RE, Kildsig DO. Effect of nicotinamide and urea on the solubility of riboflavin in various solvents. J Pharm Sci. 1996;85(9): 951-954.

39. Sanghvi R, Evans D, Yalkowsky SH. Stacking complexation by nicotinamide: a useful way of enhancing drug solubility. Int J Pharm. 2007; 336(1):35-41.

40. Suzuki H, Sunada H. Mechanistic studies on hydrotropic solubilization of nifedipine in nicotinamide solution. Chem Pharm Bull (Tokyo). 1998; 46(1):125-130.

41. Cui Y, Xing C, Ran Y. Molecular dynamics simulations of hydrotropic solubilization and self-aggregation of nicotinamide. J Pharm Sci. 2010; 99(7):3048-3059.

42. Zhang Y, Zhang K, Wu Z, et al. Evaluation of transdermal salidroside delivery using niosomes via in vitro cellular uptake I. Int J Pharm. 2015; 478(1):138-146.

43. Alvarez-Román R, Naik A, Kalia YN, Guy RH, Fessi H. Skin penetration and distribution of polymeric nanoparticles. J Control Release. 2004;99(1):53-62.

44. Jenning V, Gysler A, Schäfer-Korting M, Gohla SH. Vitamin A loaded solid lipid nanoparticles for topical use: occlusive properties and drug targeting to the upper skin. Eur J Pharm Biopharm. 2000; 49(3):211-218.

45. Campbell CS, Contreras-Rojas LR, Delgado-Charro MB, Guy RH. Objective assessment of nanoparticle disposition in mammalian skin after topical exposure. J Control Release. 2012;162(1):201-207.

46. Boakye CH, Patel K, Singh M. Doxorubicin liposomes as an investigative model to study the skin permeation of nanocarriers. Int J Pharm. 2015;489(1-2):106-116.

47. Xing J, Deng L, Li J, et al. Amphiphilic poly $\{[\alpha-$ maleic anhydride$\omega$-methoxy-poly (ethylene glycol)]-co-(ethyl cyanoacrylate) $\}$ graft copolymer nanoparticles as carriers for transdermal drug delivery. Int J Nanomedicine. 2009;4:227-232.

48. Lademann J, Knorr F, Richter H, et al. Hair follicles-an efficient storage and penetration pathway for topically applied substances. Skin Pharmacol Physiol. 2008;21(3):150-155.

49. Pople PV, Singh KK. Targeting tacrolimus to deeper layers of skin with improved safety for treatment of atopic dermatitis-part II: in vivo assessment of dermatopharmacokinetics, biodistribution and efficacy. Int J Pharm. 2012;434(1-2):70-79.

50. Pople PV, Singh KK. Development and evaluation of colloidal modified nanolipid carrier: application to topical delivery of tacrolimus, part IIIn vivo assessment, drug targeting, efficacy, and safety in treatment for atopic dermatitis. Eur J Pharm Biopharm. 2013;84(1):72-83.

51. Rancan F, Gao Q, Graf C, et al. Skin penetration and cellular uptake of amorphous silica nanoparticles with variable size, surface functionalization, and colloidal stability. ACS Nano. 2012;6(8):6829-6842. 
52. Mu CF, Balakrishnan P, Cui FD, et al. The effects of mixed MPEGPLA/Pluronic ${ }^{\circledR}$ copolymer micelles on the bioavailability and multidrug resistance of docetaxel. Biomaterials. 2010;31(8):2371-2379.

53. Dong Y, Feng SS. In vitro and in vivo evaluation of methoxy polyethylene glycol-polylactide (MPEG-PLA) nanoparticles for small-molecule drug chemotherapy. Biomaterials. 2007;28(28):4154-4160.
54. Li X, Chen D, Le C, et al. Novel mucus-penetrating liposomes as a potential oral drug delivery system: preparation, in vitro characterization, and enhanced cellular uptake. Int J Nanomedicine. 2011;6:3151-3162.

55. Zhou J, Haggerty JG, Milstone LM. Growth and differentiation regulate CD44 expression on human keratinocytes. In Vitro Cell Dev Biol Anim. 1999;35(4):228-235. 


\section{Supplementary material}

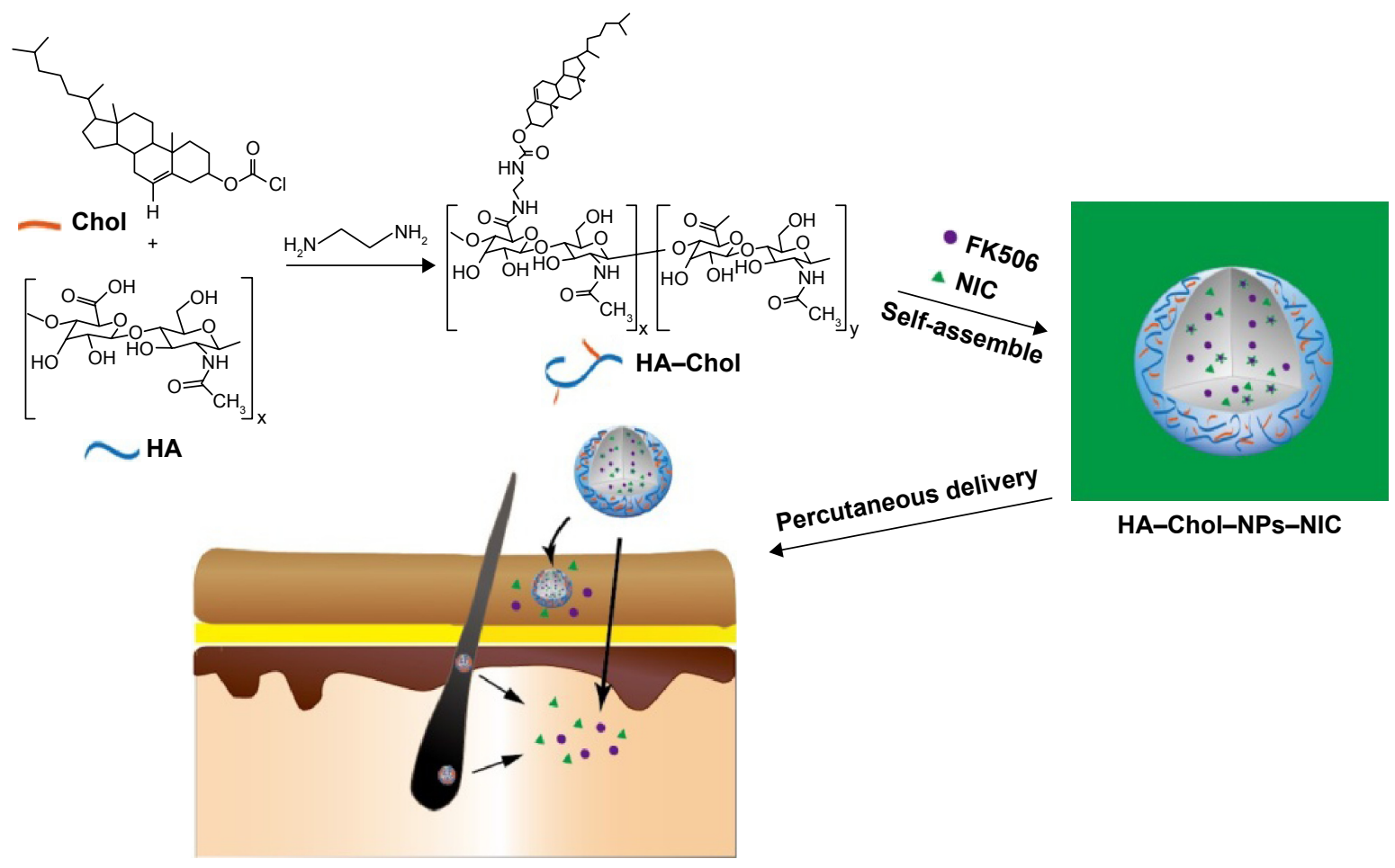

Figure SI Graphical abstract of HA-Chol nanoparticle with nicotinamide for FK506 percutaneous delivery.

Abbreviations: Chol, cholesterol; FK506, tacrolimus; HA-Chol, hyaluronic acid-cholesterol conjugates; HA-Chol-NPs-NIC, hyaluronic acid-cholesterol nanoparticles containing nicotinamide; NIC, nicotinamide.

\section{Publish your work in this journal}

The International Journal of Nanomedicine is an international, peerreviewed journal focusing on the application of nanotechnology in diagnostics, therapeutics, and drug delivery systems throughout the biomedical field. This journal is indexed on PubMed Central, MedLine, CAS, SciSearch $®$, Current Contents ${ }^{\circledR} /$ Clinical Medicine,
Journal Citation Reports/Science Edition, EMBase, Scopus and the Elsevier Bibliographic databases. The manuscript management system is completely online and includes a very quick and fair peer-review system, which is all easy to use. Visit http://www.dovepress.com/ testimonials.php to read real quotes from published authors. 